\title{
Application of a Custom Depletion Framework to the Prediction of Neutron Flux Distribution Through Depletion
}

\author{
Andrew Johnson and Dan Kotlyar \\ Georgia Institute of Technology \\ 770 State St. Atlanta, GA \\ ajohnson400@gatech.edu,dan.kotlyar@me.gatech.edu
}

\begin{abstract}
Previous works by the authors have introduced the spatial flux variation method (SFV) for predicting the changes in neutron flux due to a change in material compositions. In order to remove a full transport solution at the end-of-step, this work presents a framework responsible for computing macroscopic cross sections after a depletion event. These endof-step cross sections are estimators of changes in neutron loss and production, and enable the prediction of neutron flux using only information obtained from a single beginningof-step transport solution. The framework reads in all relevant data needed to model the depletion system, including one-group cross sections and effective fission yields to reproduce the problem using an external solver. The framework also supports extrapolating microscopic cross sections in order to rebuild the end-of-step macroscopic cross sections needed for the flux prediction. Results indicate that the SFV method is not adversely effected by the external depletion solution, and can be implemented alongside an existing transport-depletion framework.
\end{abstract}

\section{Introduction}

The field of depletion as it pertains to reactor physics is responsible for modeling isotopic compositions over time. These compositions influence the transport solutions and are tightly coupled. The traditional approach is to adopt a quasi-static approach when splitting each of the physics. The steady-state neutron transport equation is solved for a specific point in calendar time using a fixed set of compositions. Reaction rates are computed and then held constant across some time interval to obtain new compositions. In reality, the reaction rates are changing as the compositions change and thus the above approximation is only valid for very small time steps. An alternative is to use multiple transport and depletion solutions to re-evaluate the reaction rates over time.

With the intent of reducing the number of these internal transport simulations, the authors have previously presented the spatial flux variation (SFV) method for predicting changes in neutron scalar flux through depletion. The method relies on first order perturbation theory, and is fully presented in [1]. The method requires knowledge of the end-of-step macroscopic cross sections, which were previously determined using a full transport solution at the end-of-step. This removes the need for a flux prediction, as the neutron flux is already determined. 
This paper introduces a custom depletion framework for producing these macroscopic cross sections without a second transport solution. Nuclear data that is readily available to modern neutron transport codes is used to deplete materials across a depletion interval to obtain new compositions. Microscopic cross sections are extrapolated to approximate spectral changes. The result of this framework is a prediction in the neutron flux distribution using only information obtained at the beginning-of-step. The accuracy of the prediction is within a few percent of the exact flux provided by a transport solution, even in the presence of large spatial changes.

\section{Spatial Flux Variation Method}

The SFV method seeks to determine the change in scalar flux due to a general perturbation, such as changing material compositions. For completeness, a summary is presented here, in order to provide the motivation for this work. The beginning- and end-of-step transport equations are represented as

$$
L^{0} \Psi^{0}=\lambda^{0} M^{0} \Psi^{0}
$$

and

$$
L^{1} \Psi^{1}=\lambda^{1} M^{1} \Psi^{1}=\left(L^{0}+\delta L\right)\left(\Psi^{0}+\delta \Psi\right)=\left(\lambda^{0}+\delta \lambda\right)\left(M^{0}+\delta M\right)\left(\Psi^{0}+\delta \Psi\right) .
$$

The goal is to determine $\Psi^{1}=\Psi^{0}+\delta \Psi$ using first order perturbation theory and a set of complete and bi-orthogonal basis functions $\psi_{n}$ and $\psi_{n}^{\dagger}$. By representing $\Delta \Psi$ as a truncated sum of these basis functions,

$$
\delta \psi=\sum_{n=0}^{N} a_{n} \psi_{n},
$$

one can approximate the change in flux and the flux after the perturbation. The expansion coefficients $a_{n}$ are found using forward and adjoint modes of the fission matrix, as a proxy for the higher order modes of the forward and adjoint flux. However, the method also requires computing the change in the neutron loss and production operators $\delta L$ and $\delta M$. In previous works, these values were taken to be the change in macroscopic cross sections in non-overlapping spatial domains,

$$
\delta L \approx \Sigma_{a}^{1}-\Sigma_{a}^{0}
$$

and

$$
\delta L \approx \nu \Sigma_{f}^{1}-\nu \Sigma_{f}^{0}
$$

and computed by taking the exact EOS macroscopic cross sections, $\Sigma_{a}^{1}, \nu \Sigma_{f}^{1}$. Unfortunately, having the exact macroscopic cross sections implies that the exact flux distribution is also known.

This paper presents a custom depletion framework that, when attached to a transport solver, is capable of depleting each burnable material and computing the EOS macroscopic cross sections. These values are computed by obtaining new compositions through depletion and extrapolation of microscopic cross sections. Using this information, the custom depletion framework can replicate depletion results from Serpent [2] and predict the EOS flux distribution with minimal added error.

\section{Depletion Framework}

The depletion framework is built to read Serpent [2] data files and mimic the internal depletion routine. With a new set of compositions, one could obtain the EOS macroscopic cross sections in 
one of two ways. First, under the assumption that the beginning of step microscopic cross sections do not change, the macroscopic cross sections can be rebuilt with

$$
\delta \Sigma_{r}=\sum_{i} \sigma_{i, r}^{0}\left(N_{i}^{1}-N_{i}^{0}\right) .
$$

The fundamental assumption here is that the neutron spectrum not has changed dramatically such that the microscopic cross sections are constant. A more consistent model would be to determine or approximate the microscopic cross sections at the next depletion step and compute

$$
\delta \Sigma_{r}=\sum_{i}\left(\sigma_{i, r}^{1} N_{i}^{1}-\sigma_{i, r}^{0} N_{i}^{0}\right)
$$

Therefore the goal of this framework is to obtain two EOS quantities: new compositions, $\left\{N_{i}^{1}\right\}$ and new microscopic cross sections $\left\{\sigma_{r, i}^{1}\right\}$. These tasks are discussed in Sections 3.1 and 3.2.

\subsection{Solution of depletion equations}

Determining the updated compositions of burnable materials involves solving the following coupled ordinary differential equation

$$
\frac{\partial \vec{N}}{\partial t}=\boldsymbol{A} \vec{N}(t)
$$

given initial compositions $\vec{N}(0)=\vec{N}_{0}$. The matrix $\boldsymbol{A}$ is a square matrix containing isotopic decay and transmutation rates to and from other isotopes. Detailed information on the nature and structure of the transmutation matrix $\boldsymbol{A}$ can be found in [3]. The solution for Eq. 6 after some $\Delta t$ can be formally expressed as

$$
\vec{N}(t+\Delta t)=\exp (\boldsymbol{A} \Delta t) \vec{N}(t) .
$$

Solving Eq. 7 involves taking the exponent of matrix $\boldsymbol{A}$, which can become very stiff, with values ranging from $O\left(10^{ \pm 17}\right)$. Furthermore, the main diagonal of the matrix describes the destruction of isotopes and is entirely negative, while off-diagonal elements are positive for production. The incomplete partial factorization (IPF) form of the Chebyshev Rational Approximation Method (CRAM) [4] was used in this work to solve Eq. 7 for all burnable materials. This approach is well suited for burnup problems, and is used in the Serpent depletion routines.

The depletion framework consists of a collection of Python objects representing each burnable material. Each object contains isotopic compositions and representation of decay and transmutation chains. This structure, in conjunction with one-group cross sections and scalar flux, allows the computation of reactions rates and construction of the full depletion matrix. The IPF implementation relies heavily on numpy and scipy $[5,6]$.

\subsection{Extrapolation of microscopic cross sections}

Each burnable material object also stores microscopic reaction cross sections for the beginning-ofstep (BOS) and, optionally, for some number of previous steps. Simple polynomial fitting yields a set of coefficients needed to approximate the microscopic cross sections across the depletion interval $\Delta t$. Microscopic absorption and fission cross sections are needed to evaluate $\delta L$ and $\delta M$, while other reaction cross sections are stored to rebuild $\boldsymbol{A}$ for the BOS depletion event. Lastly, the 
average number of neutrons per fission $\bar{\nu}$ was also extrapolated to properly estimate $\nu \Sigma_{f}^{1}$, but this quantity is homogenized across the burnable material.

\section{Results}

The custom depletion framework was tested using a PWR fuel pin with realistic axial moderator profile from [1]. The Serpent Monte Carlo code was used to deplete out to 60 days using five depletion schedules. All started with ten one-day steps to model fission product production. The remaining 50 day interval was divided into $1,2,5,25$, and 50 intervals of 50, 25, 10, 2, and 1 day steps, respectively. The SFV method was responsible for predicting the flux at day 60 using information gathered from the penultimate step, e.g. day 35 using 25 day steps, day 59 using 1 day steps, etc. The problem was divided into 10 axial regions with macro- and microscopic cross sections extracted at each depletion step using Serpent.

First, the IPF solver was compared to Serpent's internal routines by solving Eq. 6 using the same initial compositions and depletion matrix. Figure 1 plots the difference in final EOS atom densities as computed by the depletion framework and the Serpent EOS atom densities. The x-axis is the actual EOS density provided by Serpent in the depletion matrix files. This figure demonstrates that, for the step sizes considered in this work, the external IPF solver is an acceptable replacement for the internal Serpent routine. The largest absolute errors are 12 orders of magnitude less than the final compositions $\left(\Delta N=10^{-15} \# / b-c m\right.$ of $\left.10^{-2} \# / b-c m\right)$. Larger relative errors exist for trace isotopes $\left(N \leq 10^{-15} \# / b-c m\right)$, many of which do not have cross section data.

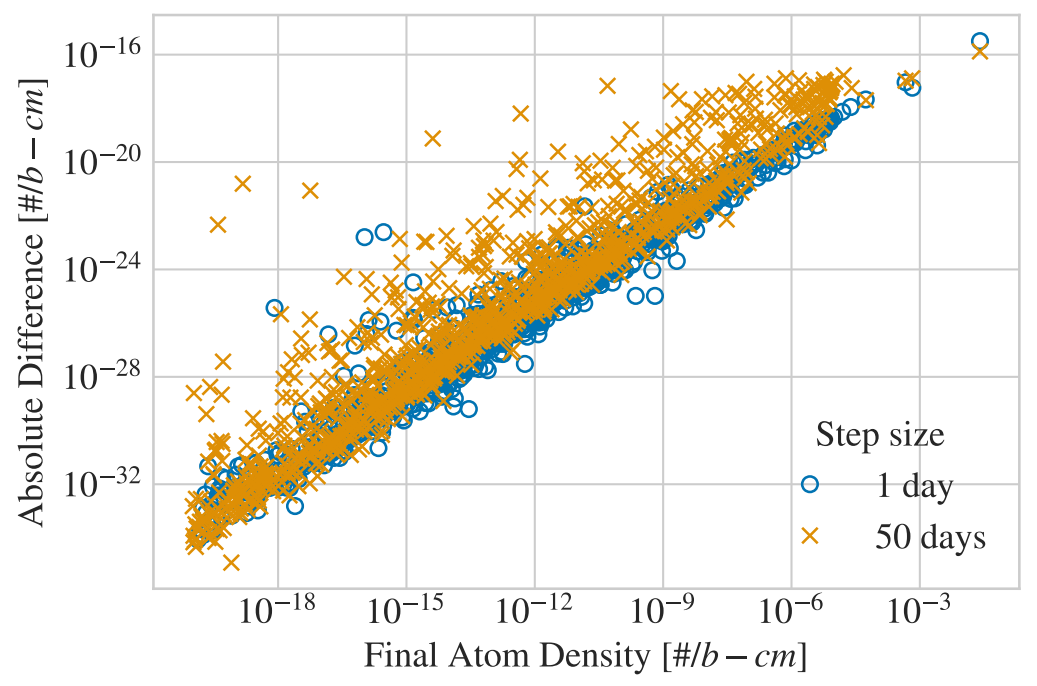

Figure 1: Absolute difference in EOS compositions with external IPF solver compared to Serpent internal solver

Having obtained the new compositions at the end-of-step (EOS), the next task is to determine the new microscopic cross sections to evaluate EOS macroscopic cross sections using Eq. 5. Important reactions for typical thermal spectrum problems are highlighted in Fig. 2. After inspecting Fig. 2, it 
was determined that linear extrapolation would be sufficient to predict the evolution in microscopic cross sections.

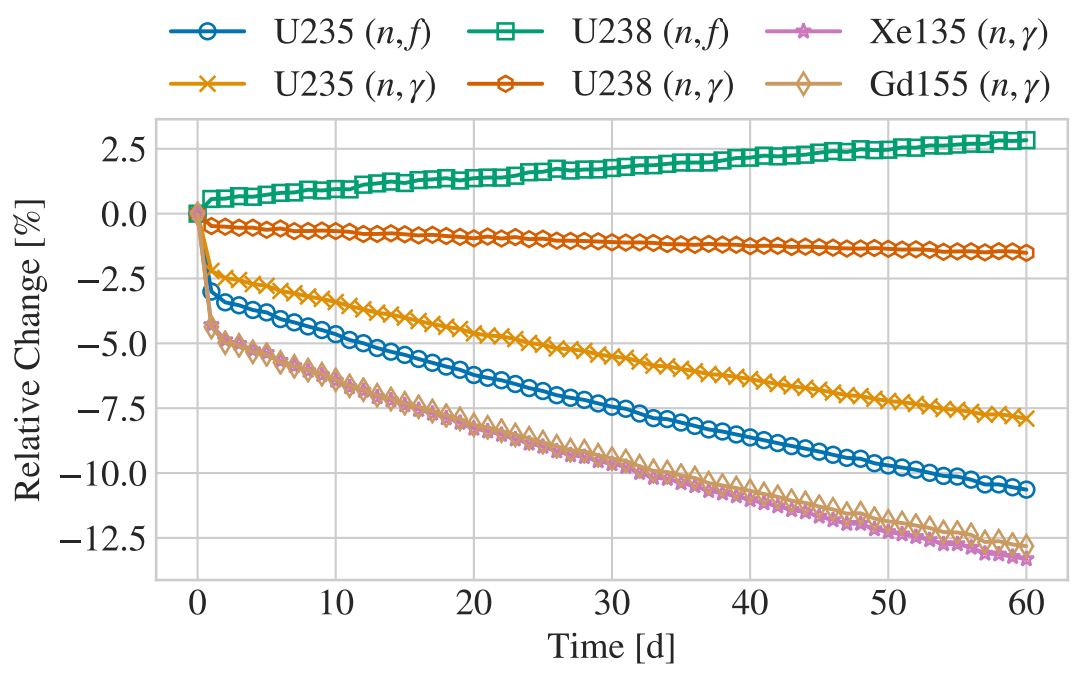

Figure 2: Relative change in microscopic cross sections for select reactions in a single region

Figure 3 presents a wider distribution of errors using the external depletion solver and the custom depletion framework. Every burnable material was depleted across all depletion steps and compared to the expected EOS composition provided by Serpent. The depletion matrices $\boldsymbol{A}$ were computed using BOS microscopic cross sections returned from Serpent. The absolute difference in number density was multiplied by the BOS microscopic absorption cross section to estimate the contribution of errors to macroscopic absorption cross section. For this specific problem, the macroscopic absorption cross section for each material was on the order of $10^{-2} \mathrm{~cm}^{-1}$, meaning the errors in Fig. 3 constitute less than $0.05 \%$.

Using the external depletion solver and custom framework, the spatial flux variation method [1] was used to determine the final flux distribution. 20 expansion coefficients were used when computing the change in flux between two points. Figure 4 contains two sets of flux data taken from the simulation using two different depletion steps. First, the beginning- and end-of-step distributions are plotted using 25 and 50 day steps. The dashed lines represent the flux prior to the final depletion event computed at day 35 and day 10, respectively. The solid line denotes the flux at day 60 for both step sizes, and the distributions are markedly different.

The other quantities plotted are the flux predicted by the SFV method, first using exact EOS macroscopic cross sections and then using the custom depletion framework. In the presence of large shifts in spatial power distribution, the SFV method, with and without the external depletion framework, is able to capture the EOS flux to within a few percent. The RMS error is presented in Table 1 comparing the SFV predicted fluxes to those generated by Serpent. The BOS column serves to quantify the change in flux distribution across each of the depletion steps. Since the custom depletion framework is fitting the macroscopic cross sections, while the exact case is using the true end-of-step macroscopic cross sections, improvements found with the custom framework are likely 

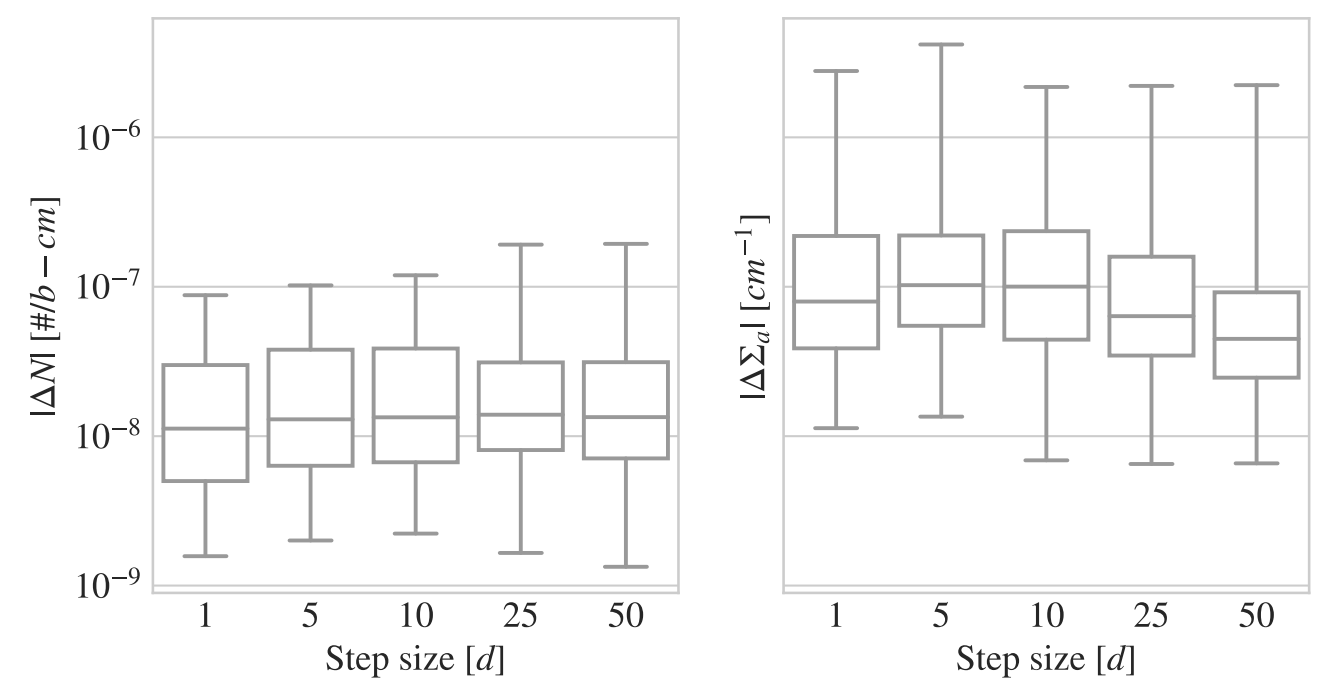

Figure 3: Distribution of errors in predicted number densities (left) and macroscopic absorption cross section (right) using custom depletion framework

attributable to beneficial error cancellation.

Table 1: RMS Errors for BOS and SFV fluxes

\begin{tabular}{lrrr}
\hline Step size $[\mathrm{d}]$ & BOS & SFV - Exact $\Sigma$ & SFV - Custom \\
\hline 1 & $2.589 \mathrm{E}-03$ & $1.595 \mathrm{E}-03$ & $1.934 \mathrm{E}-03$ \\
5 & $1.384 \mathrm{E}-03$ & $9.608 \mathrm{E}-04$ & $1.176 \mathrm{E}-03$ \\
10 & $2.401 \mathrm{E}-03$ & $1.105 \mathrm{E}-03$ & $1.251 \mathrm{E}-03$ \\
25 & $8.750 \mathrm{E}-03$ & $3.068 \mathrm{E}-03$ & $2.417 \mathrm{E}-03$ \\
50 & $3.567 \mathrm{E}-02$ & $5.207 \mathrm{E}-03$ & $5.084 \mathrm{E}-03$ \\
\hline
\end{tabular}

To compare the efficiency of the SFV method, a figure of merit is presented that weights the time saved to square of RMS error

$$
F O M=\frac{S F}{R M S^{2}} .
$$

As a comparison, the predicted EOS flux from the SFV method will be compared as if one had taken the BOS flux to be the EOS flux. In doing this, the speed up factor, $S F$ will be 2, as one transport solution is avoided. Since the SFV method relies upon a more expensive transport solution to generate the fission matrix, but avoids the EOS transport solution when using the coupled depletion framework, the speed up factor used will be the cost of computing the fission matrix for this problem. Across all depletion steps and schemes, the average runtime increase $T_{f m} / T_{r e f}$ was 1.15 . 

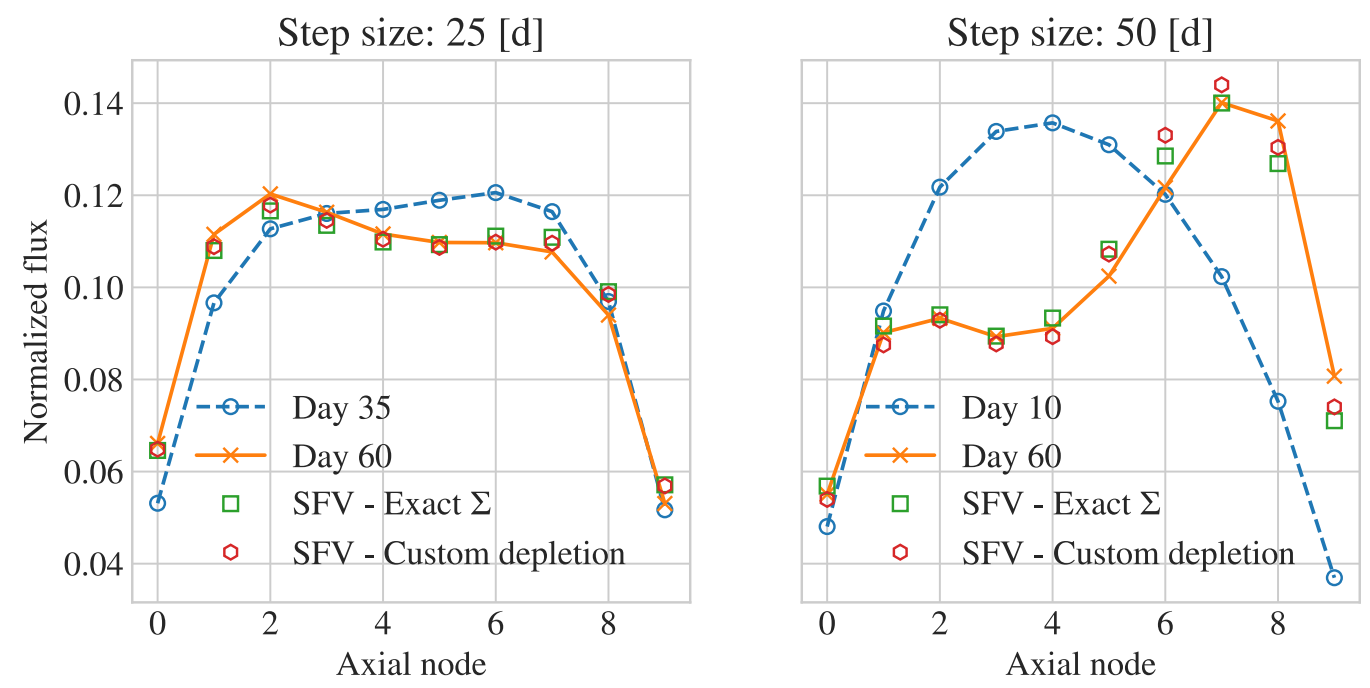

Figure 4: BOS and EOS Serpent fluxes for 25 and 50 day steps with SFV predicted fluxes

The ratio of SFV to BOS figures of merit is plotted in Fig. 5. For smaller step sizes, the flux distribution does not change substantially, as indicated in Table 1. Therefore for smaller steps the SFV method does not merit the additional computational cost. However, for larger depletion steps the SFV method greatly out performs as it is able to capture large variations in spatial flux with a modest performance penalty.

\section{Conclusion}

A custom depletion framework was written to study how the spatial flux variation (SFV) method would behave in a practical setting. This is done to approximate the end-of-step (EOS) macroscopic cross sections without running a full transport simulation that could easily return the desired flux, negating the need for a prediction. The depletion framework uses information that is readily available using modern neutron transport codes: decay and transmutation chains taken from nuclear data files, one group cross sections easily computed with tallies, and homogenized macroscopic cross sections.

The framework was validated by comparing the internal depletion routines to identical routines used by Serpent. Macroscopic cross sections were computed by obtaining new compositions with a custom CRAM solver, and extrapolating microscopic cross sections. These macroscopic cross sections were used to predict the EOS flux distribution using the SFV method. After comparing this flux to a Serpent simulation and the predicted flux using exact EOS macroscopic cross sections, the custom depletion framework was found to introduce no substantial errors.

This work demonstrates that the SFV method is a promising way to predict how the spatial flux distribution changes following a depletion event. By removing the need to exactly know the EOS macroscopic cross sections, the method can be integrated along side a coupled transport-depletion routine. Since almost all of the information can be readily obtained from within a transport simu- 


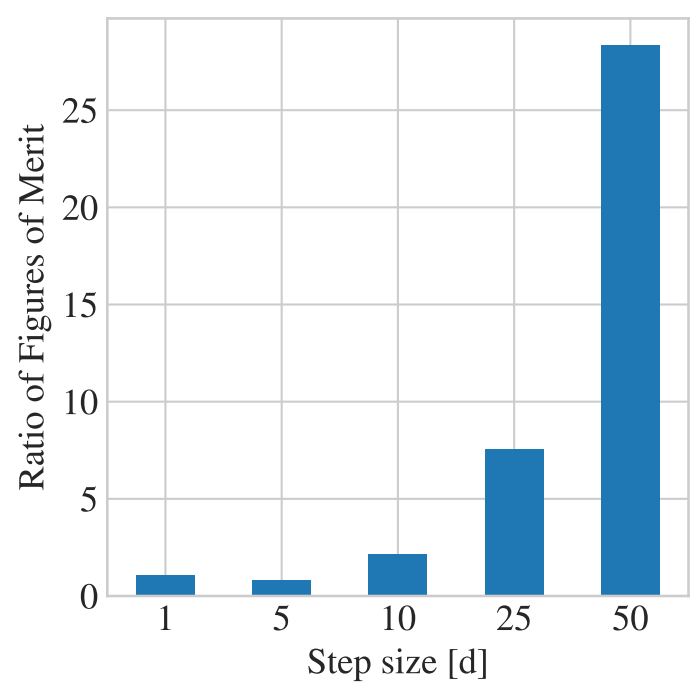

Figure 5: Ratio of custom depletion to BOS figures of merit

lation, the only major performance reduction is from the fission matrix. For larger problems, this will become a non-negligible penalty and thus alternative methods are under consideration. Furthermore, as the microscopic cross sections are able to be extrapolated well, the custom depletion framework is being explored as a sub-step solution, where the flux will be predicted through a depletion interval. This predicted sub-step flux can be used to deplete the materials, in concert with extrapolated microscopic cross sections, to approach a continuous-in-time depletion solution.

\section{REFERENCES}

[1] A. E. Johnson and D. Kotlyar. "A Transport-Free Method for Predicting the Post-depletion Spatial Neutron Flux Distribution.” Nuclear Science and Engineering, volume 194, pp. 120 137 (2020).

[2] J. Leppänen, M. Pusa, T. Viitanen, V. Valtavirta, and T. Kaltiaisenaho. "The Serpent Monte Carlo Code: Status, development, and applications in 2013." Annals of Nuclear Energy, volume 82, pp. 142-150 (2015).

[3] M. Pusa and J. Leppänen. "Computing the Matrix Exponential in Burnup Calculations.” Nuclear Science and Engineering, volume 164(2), pp. 140-150 (2010).

[4] M. Pusa. "Higher-Order Chebyshev Rational Approximation Method and Application to Burnup Equations." Nuclear Science and Engineering, volume 182(2), pp. 297-318 (2016).

[5] Stéfan van der Walt, S. C. Colbert, and G. Varoquaux. "The NumPy Array: A Structure for Efficient Numerical Computation." Computing in Science \& Engineering, volume 9, pp. 2129 (2007).

[6] E. Jones, T. Oliphant, P. Peterson, et al. "SciPy: Open source scientific tools for Python." (2001-). URL http://www.scipy.org/. [Online]. 